\title{
Decomposing Magnetic Dark-Field Contrast in Spin Analyzed Talbot-Lau Interferometry: A Stern-Gerlach Experiment without Spatial Beam Splitting
}

\author{
Jacopo Valsecchi® \\ Laboratory for Neutron Scattering and Imaging, Paul Scherrer Institut, Villigen, Switzerland and University of Geneva, Switzerland \\ Malgorzata G. Makowska® \\ Photons for Engineering and Manufacturing Group, Paul Scherrer Institut, Villigen, Switzerland \\ Youngju Kim and Seung Wook Lee 1 \\ School of Mechanical Engineering, Pusan National University, Busan, South Korea \\ Christian Grünzweig \\ Laboratory for Neutron Scattering and Imaging, Paul Scherrer Institut, Villigen, Switzerland \\ Florian M. Piegsa \\ Laboratory for High Energy Physics, Albert Einstein Center for Fundamental Physics, University of Bern, Bern, Switzerland \\ Michel A. Thijs $\odot$ and Jeroen Plomp \\ Faculty of Applied Sciences, Delft University of Technology, Delft, Netherlands \\ Markus Stroblø* \\ Laboratory for Neutron Scattering and Imaging, Paul Scherrer Institut, Villigen, Switzerland and \\ Nils Bohr Institute, University of Copenhagen, Copenhagen, Denmark
}

(Received 23 April 2020; revised 7 November 2020; accepted 6 January 2021; published 18 February 2021)

\begin{abstract}
We have recently shown how a polarized beam in Talbot-Lau interferometric imaging can be used to analyze strong magnetic fields through the spin dependent differential phase effect at field gradients. While in that case an adiabatic spin coupling with the sample field is required, here we investigate a nonadiabatic coupling causing a spatial splitting of the neutron spin states with respect to the external magnetic field. This subsequently leads to no phase contrast signal but a loss of interferometer visibility referred to as dark-field contrast. We demonstrate how the implementation of spin analysis to the Talbot-Lau interferometer setup enables one to recover the differential phase induced to a single spin state. Thus, we show that the dark-field contrast is a measure of the quantum mechanical spin split analogous to the Stern-Gerlach experiment without, however, spatial beam separation. In addition, the spin analyzed dark-field contrast imaging introduced here bears the potential to probe polarization dependent small-angle scattering and thus magnetic microstructures.
\end{abstract}

DOI: 10.1103/PhysRevLett.126.070401

The peculiar feature of neutrons as charge neutral particles carrying a magnetic moment has been exploited in the study of magnetic phenomena [1-17]. In neutron imaging, the interaction of the neutron magnetic moment with magnetic fields is exploited in various ways to image macroscopic magnetic fields and structural features [9,18-29].

Nonadiabatic spin interaction with an external magnetic field can be expressed in a quasiclassic description by a rotation of the neutron magnetic moment $\boldsymbol{\mu}_{n}$ around the magnetic field $\boldsymbol{B}$ according to the Bloch equation [30,31]

$$
\frac{d \boldsymbol{\mu}_{n}}{d t}=\gamma_{n} \boldsymbol{\mu}_{n} \times \boldsymbol{B}
$$

referred to as Larmor precession, where $t$ is time and $\gamma_{n}=2 \mu_{\mathrm{n}} / \hbar$. The Larmor frequency is $\omega_{L}=\gamma_{n} B$.
The interaction of an unpolarized beam with structures like magnetic domain walls leaves a signature in darkfield contrast images (DFIs) and enables one to observe the domain structure in the volume of bulk samples [32-39].

DFIs are based on the local loss of visibility in a Talbot-Lau grating interferometer [40-42]. A Talbot-Lau interferometer consists of three gratings, as depicted in Fig. 1(a). The source grating $G_{0}$ creates the partial coherence that enables the formation of the interference pattern induced by the phase grating $G_{1}$ and the analyzer grating $\mathrm{G}_{2}$ at the $n$th fractional Talbot distance [43]. The technique can provide a threefold contrast related to the parameters describing the interference pattern $I$ that can be recorded in every pixel of an image as [40] 


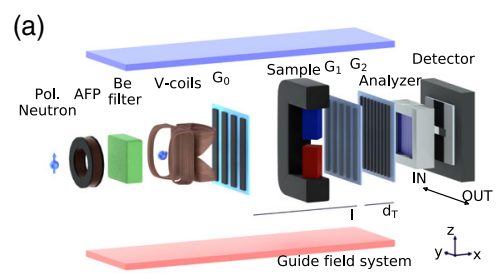

(b)

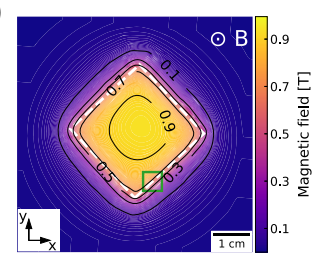

FIG. 1. Setup details. (a) Sketch of the polarized Talbot-Lau interferometer including optional polarization analysis. The setup consists of an adiabatic fast passage spin flipper (AFP), a beryllium filter (Be filter), two V-coils, a source grating $G_{0}$, a phase grating $G_{1}$, an analyzer grating $G_{2}$, a detector, a guide field system, and a polarization analyzer. The probed polarization direction is along the $z$ axis. The sample is placed between $\mathrm{G}_{0}$ and $G_{1}$. The North and South poles are depicted in red and blue. (b) Hall probe map of the magnetic field between the squareshaped pole shoes. The white line indicates the edges of the magnets, while the green one highlights the region at which the effect is described by the Wigner function calculations.

$$
I(s)=a+b \cos \left(2 \pi \omega_{s} s+C_{r}\right)
$$

where $s$ is the transversal scanning parameter of one grating, $a$ is the offset, and $a / a_{0}$ represents the attenuation contrast where the index 0 refers to the undisturbed interference. $(b / a) /\left(b_{0} / a_{0}\right)$ provides the DFI, which is the relative visibility $V / V_{0}$, where $V=\left(I_{\max }-I_{\min }\right) /\left(I_{\max }+I_{\min }\right)$, with $I_{\max }$ and $I_{\min }$ being the modulation intensity at maximum and minimum values [41]. $C_{r}$ is the relative phase of the interference modulation compared to the undisturbed pattern $\left(C_{0}=0\right)$. It measures the local differential phase of the neutron wave, allowing one to observe the refractive index distribution. $\omega_{s}=1 / p$ represents the inverse period $p$ of the interference pattern.

DFIs have been found to be capable of detecting microscopic structures through the small-angle scattering they induce to the beam. While initially only varying structural features could be detected, today scanning of the probed correlation length defined by $\xi=\lambda L_{s} / p$, where $\lambda$ is the wavelength and $L_{s}$ is the sample to $\mathrm{G}_{2}$ distance, paired with corresponding analysis, provides quantitative microstructural information [44]. The potential for obtaining spatially resolved information about bulk magnetic structures proved unique and valuable in the investigation of applied materials. The achieved contrast has so far been explained by an induced loss of coherence, while we here demonstrate that it is in fact generated by an increasing degree of destructive superposition of intensities rather than a coherence dependent destructive phase superposition [41].

Recently, we have shown that the use of a polarized neutron beam in a Talbot-Lau interferometer enables the characterization of strong magnetic fields through differential phase contrast imaging (DPCI) in cases where the incident spin couples adiabatically to the probed magnetic field [29]. Such an adiabatic transition takes place when
$\omega_{L} \gg(\delta \boldsymbol{B} /|\boldsymbol{B}|) / \delta t$, where $\delta \boldsymbol{B} /|\boldsymbol{B}|$ indicates the changes in direction of $\boldsymbol{B}$ [5].

The Pauli equation implies the coupling of spin and momentum and for a free neutron in an external magnetic field one can write the time independent Hamiltonian:

$$
\mathcal{H}=\frac{p^{2}}{2 m_{n}}-\boldsymbol{\mu}_{\boldsymbol{n}} \cdot \boldsymbol{B}=\frac{\hbar^{2} k^{2}}{2 m_{n}}-\boldsymbol{\mu}_{\boldsymbol{n}} \cdot \boldsymbol{B},
$$

where $p$ is the momentum operator, with $k=2 \pi / \lambda$ and the neutron magnetic moment operator defined as $\boldsymbol{\mu}_{\boldsymbol{n}}=\mu_{n} \boldsymbol{\sigma}$, with $\sigma$ being the spin operator. The energy conservation demands the neutron with an initial kinetic energy matching the total energy $E_{\text {tot }}=E_{k 0}=\hbar^{2} k_{0}^{2} / 2 m_{n}$ to gain or lose momentum corresponding to the gained or lost potential energy corresponding to [5]

$$
E_{\mathrm{tot}, B}=E_{k}=\frac{\hbar^{2} k_{0}^{2}}{2 m_{n}} \pm \mu_{n} B=E_{k 0} \pm \Delta E_{k} .
$$

Note that for $\Delta E_{k}$, the orientations of the magnetic moment and $B$ are not relevant because the relevant spin-up and spindown states are oriented parallel $|\uparrow\rangle$ and antiparallel $|\downarrow\rangle$ to the applied field $B$. The implied shift in kinetic energy of the neutron contributes to the refractive index [18]

$$
\delta_{\mu}= \pm \frac{\mu_{n} B}{2 E_{k 0}}= \pm \frac{2 m_{n} \lambda^{2} \mu_{n} B}{h^{2}}
$$

In the presence of a magnetic field gradient, a differential phase effect is measured, where the \pm refers to the Zeeman splitting.

Here, we consider the transversal splitting of the spin states of the beam polarized perpendicular to a prismshaped external magnetic field to which the neutron beam polarization is nonadiabatically coupled $[45,46]$. We apply the setup used in Ref. [29], with a polarized incident beam and a vertical guide field throughout the grating interferometer setup and a well-defined vertical magnetic field with a square cross section oriented at $45^{\circ}$ to the beam in the sample position between $\mathrm{G}_{0}$ and $\mathrm{G}_{1}$, as shown in Fig. 1 . The initial vertical polarization is turned into the horizontal plane and an optional polarization analyzer has been added between the analyzer grating $\mathrm{G}_{2}$ and the detector, which does not disturb the interference and introduces only a minor impact on the spatial resolution. Upon transmission of the sample field, oriented antiparallel to the guide field, the transversal gradient of the energetic and thus the longitudinal separation of the spin states will result in an additional transversal separation of the spin-up and spindown states. While the precession of the spin around the perpendicular field vector is easily measured, as routinely done in spin-echo measurements, the corresponding spatial separation of the quantum mechanically 
postulated perpendicular spin states does not lead to a sufficient beam splitting to be probed. The beam dimensions used are on the order of centimeters, while the theoretical values for the beam splitting are on the order of micrometers. We introduce a novel measurement of the spatial splitting of the spin states in a precession field despite using macroscopic beam dimensions. This resembles a Stern-Gerlach experiment but in a precession field without beam splitting.

The polarized neutron grating setup used, shown in Fig. 1(a), featured gratings with periods of $p_{0}=824 \mu \mathrm{m}$, $p_{1}=7.96 \mu \mathrm{m}$, and $p=4 \mu \mathrm{m}$ for $\mathrm{G}_{0}, \mathrm{G}_{1}$, and $\mathrm{G}_{2}$, respectively [29]. The distance $l=\left(p_{0} / p_{2}\right) /\left(d_{T}\right)$ between source and phase gratings was chosen according to the geometric constraint for constructive superposition at the first fractional Talbot distance $d_{T}=19.6 \mathrm{~mm}$. The beam is quasimonochromatic with an effective wavelength of $5.35 \AA$ and a spread $\Delta \lambda / \lambda$ of about $20 \%$, which is achieved by a cooled beryllium filter installed upstream of the interferometer in the polarized cold neutron beam of the Beam Line for Neutron Optics and Other Approaches of the Swiss Spallation Neutron Source at Paul Scherrer Institut [47]. The beam is polarized already in the extraction section of the beam line through a polarizing bender along the vertical direction, with a polarization measured to be around $90 \%$ up to the detector position with guide fields in place. An adiabatic fast passage spin flipper (AFP) is added upstream of the Be filter, and two V-coils are arranged upstream of $\mathrm{G}_{0}$. In order to avoid an adiabatic spin transition, the initial vertical polarization is turned into the horizontal plane by a double V-coil arrangement. The first V-coil turns the polarization adiabatically into the $x y$ plane through a gradually increasing horizontal field superimposed to the weak guide field. Subsequently, the second V-coil generates a sudden nonadiabatical field transition to a vertical orientation, smoothly transitioning into the vertical guide field. The optional polarization analyzer between $\mathrm{G}_{2}$ and detector was a solid state bender-type analyzer consisting of $120 \mu \mathrm{m}$ thick coated Si lamellae and aligned along the $z$ axis [48]. Images were recorded using a digital camera [Andor iKonM, $1024 \times 1024$ pixels] that recorded, via a mirror, the light from a $200 \mu \mathrm{m}$ thick $\mathrm{Li} / \mathrm{ZnS}$ scintillator screen facing the neutron beam downstream of the analyzer grating and providing an intrinsic spatial resolution of about $200 \mu \mathrm{m}$ as characterized with a Siemens star test object.

For image acquisition, a phase-stepping approach was adopted, and 21 images scanning one modulation period were recorded with an exposure time of $60 \mathrm{~s}$ for each. Images were recorded for two opposite incident spin orientations achieved by the AFP, and all resulting interferometric images of attenuation, phase, and visibility were reconstructed with the software TAPY $[29,49]$.

Initial images were taken without the polarization analyzer (Fig. 2). In contrast to the case of adiabatic coupling, no opposite differential phase is measured on the two prismatic sides of the inclined squared sample field, but a significant dark-field contrast is recorded on both sides [29]. The measured dark-field contrast is quantified to $V / V_{0}=0.87$ in the plateau regions. In the center and at the edges, this value tends toward one, which at the sample edges can be assumed to be due to spatial resolution, while in the center it indicates a smooth bowing of the field boundary in contrast to a sharp edge. No differential phase contrast is measured throughout the field region, as the phase of the interference $C_{r}$ is zero.

A second measurement is performed with the polarization analyzer. The polarization analyzer is aligned in a vertical configuration that only transmits spin-up neutrons, as depicted in Fig. 1(a). In this case, full visibility $V / V_{0} \simeq 1$ is regained, within the limits of accuracy. In contrast to the first measurement, a significant phase contrast is recorded. A constant but opposite phase shift is clearly observed at the two sides of the prism configuration. The shift $C_{r}$, again in the plateau regions, can be quantified to be $\pm 0.5 \mathrm{rad}$, as shown in Fig. 3(c), 3(d). In the interference pattern, this corresponds to a spatial shift of $0.318 \mu \mathrm{m}$. This value again smoothly transitions to zero at the edges and at the center. The average intensity is half compared to the initial measurement, which corresponds well with the removal of neutrons with opposite spin states and the assumption of an equal distribution between both.

These results appear compatible with the quantum mechanical expectation of a transversal split of the spinup and spin-down states. It can be assumed that both spin states create independent interference patterns at the fractional Talbot distance, where $\mathrm{G}_{2}$ is positioned. These interference patterns exhibit a spatial phase shift according to their differential phase. The second measurement, where one spin state is suppressed, and thus the corresponding phase shift of the other is measured, underlines this situation. In the first measurement, however, the superposition of the two separate intensity modulations for the two separate spin states, with symmetrically opposite phase offsets, is suitable for explaining the visibility loss through additive superposition of the two independent intensities represented by the DFI in the field region. A quantitative estimate considering the magnetic sample, depicted in Fig. 1(b), is in good agreement with such consideration of a superposition of two equal, separate intensity modulations corresponding to the Talbot-Lau interference. The interferometer phase shift $C_{r}$ corresponding to magnetic refraction can be calculated according to $\tan \left(\phi_{ \pm}\right)=\left(C_{r} / d_{T}\right)$, where $d_{T}$ is the partial Talbot distance, $p$ is the period of $\mathrm{G}_{2}$, and $\phi_{ \pm}=\delta \varphi_{ \pm} / \delta y$ is the refraction angle transverse to the beam introduced by the magnetic spin dependent phase shift $\varphi_{ \pm}=\int \delta_{\mu} d x_{p}$ based on the refractive index [Eq. (5)] $[29,43]$.

The calculations yield a phase shift of $C_{r}= \pm 0.5 \mathrm{rad}$, as depicted in Fig. 3(c), which agrees with the outcome of the second measurement, where this shift is directly measured for an individual spin state. This corresponds to a 
(a)

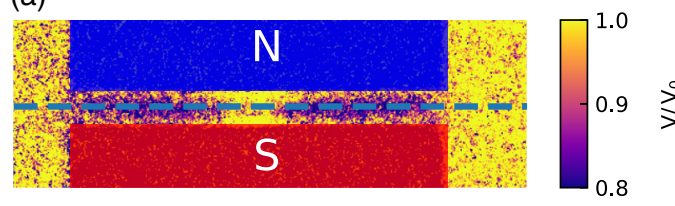

(c)

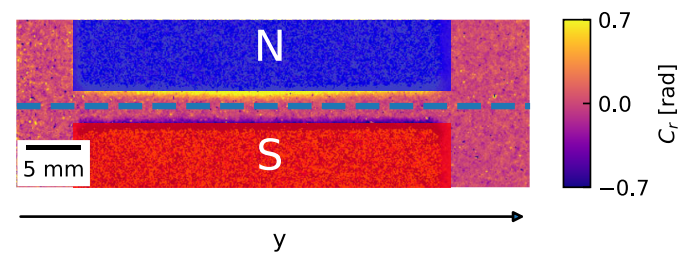

(b)
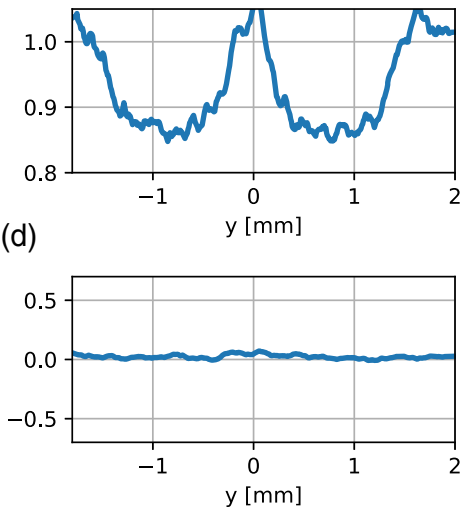

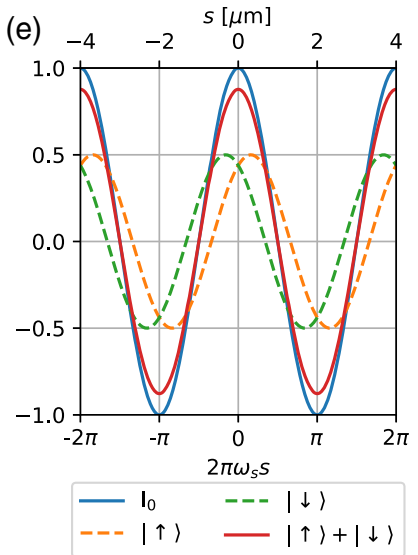

FIG. 2. Results without polarization analysis. (a) DFI and (c) DPCI of the magnetic field prism measured without the spin analyzer. The North and South pole shoes are depicted in blue and red; (a),(c) share the same size scale bar. (b),(d) Horizontal line profiles of the DFI and DPCI along the dashed lines depicted in (a),(c). (e) Illustration of the effect on the as-measured interference pattern $|\uparrow\rangle+|\downarrow\rangle$ (red) according to an ideal open beam modulation $\left(I_{0}\right)$ (blues) and the thus-assumed individual and separate interference patterns of the spin-up $|\uparrow\rangle$ (orange) and spin-down $|\downarrow\rangle$ (green) states in the plateau regions where the dark-field contrast is quantified to $V / V_{0}=0.87$.

transversal split of the spin states of $0.64 \mu \mathrm{m}$ at $\mathrm{G}_{2}$ according to a refraction angle of $16 \mu \mathrm{rad}$ and the effective sample to a $\mathrm{G}_{2}$ distance of $2 \mathrm{~cm}$. In accordance with the assumed intensity superposition probed in the first measurement, this calculation returns a relative visibility of $V / V_{0}=0.87$ [Fig. 2(b)] in agreement with the measurement result. The superposition of the two symmetrically but oppositely shifted interference patterns implies a vanishing resulting phase shift in accordance with the measurement [Fig. 2(c)]. It was demonstrated that the dark-field contrast modality of the grating interferometric neutron imaging can resolve the lateral split of spin states aligned parallel and antiparallel to a prismatic precession field. Thus, the performed dark-field measurement resembles a SternGerlach experiment in which a transversal split of spin states, on the order of a micrometer, is detected without actual beam splitting of a centimeter sized beam. It was further shown that the addition of a polarization analyzer to a polarized neutron grating interferometer enables one to decompose the dark-field signal into the differential phase contrast signatures of the individual spin states according to their deflection by the magnetic prism. Their wave functions independently create interference patterns, which are offset in phase due to the interaction with the magnetic field. It was also found that the individual wave functions of the split spin states have conserved coherence and display no visibility loss within the accuracy of the measurement. In conclusion, these results imply that the dark-field contrast, the loss of visibility, has to be assigned to a superposition of intensities rather than an interference with (a)

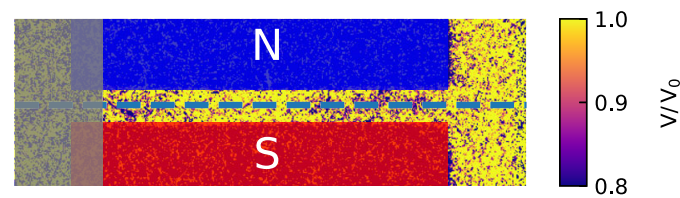

(c)

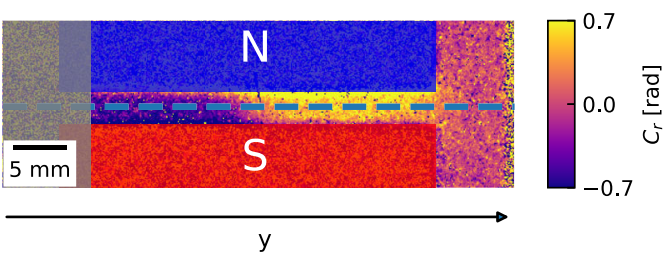

(b)

(d)
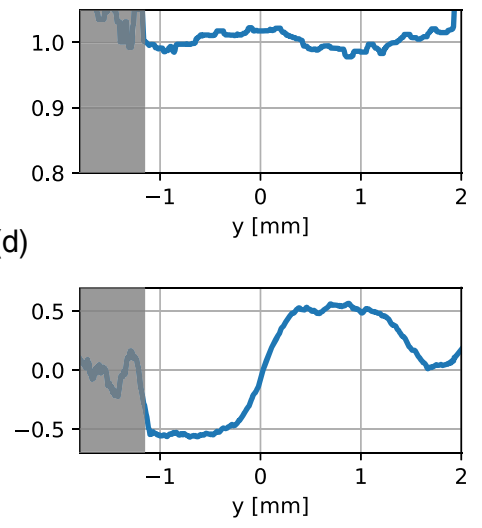

(e)

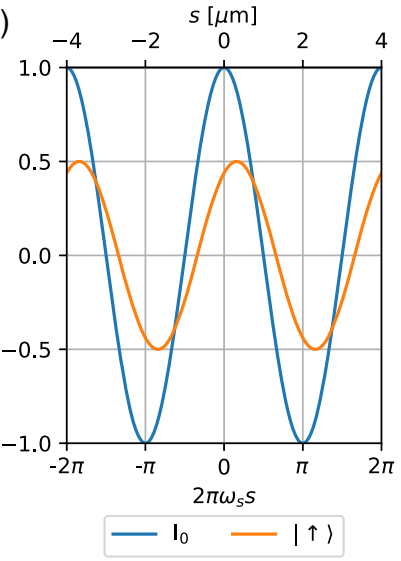

FIG. 3. Results with polarization analysis. (a) DFI and (c) DPCI of the magnetic field prism measured with the spin analyzer. The North and South pole shoes are depicted in blue and red; (a),(c) share the same size scale bar. (b),(d) Horizontal line profiles of the DFI and DPCI along the dashed lines depicted in (a),(c). The gray areas show the blind region lying outside the field of view of the analyzer. (e) Illustration of the corresponding effect on the as measured interference pattern of the spin-up $|\uparrow\rangle$ (orange), according to an ideal open beam modulation (blue) in the plateau regions where $C_{r}=0.5 \mathrm{rad}$. 
diminished coherence as has to date been argued in the literature $[32,36,39]$. The introduced setup additionally bears the potential to enable one to measure not only the spatial split of the spin-up and spin-down wave functions but simultaneously also the interference of these, which manifests in a spin precession in the perpendicular plane. Calculations suggest (see the comparison of the calculated Wigner functions in the Supplemental Material [50]) that for higher applied wavelength resolutions, e.g., around $1 \%$, which is common for many measurements, that spin precession is preserved for a significant region from the prism edge. The spatial period of the precession, with the given parameters of about $25 \mu \mathrm{m}$, is clearly resolvable with state-of-the-art spatial resolution capabilities. Thus, an optimized setup, with the polarization analyzer turned by $90^{\circ}$ compared to our setup, would provide the unique opportunity to measure simultaneously the dark-field contrast of the transversal beam splitting and the spatial modulation of the spin precession. In the field of direct observations of magnetic domains, the setup enables the simultaneous visualization of domain walls by dark-field contrast imaging [32-39] and the measurement of the domain magnetization and orientation by polarization contrast [9,20-24,28,31]. Additionally, the instrumentation can be used to extend spatially resolved quantitative smallangle scattering studies performed with dark-field contrast imaging $[41,44]$ to magnetic materials and structures by probing all components of magnetic scattering.

We gratefully acknowledge S. Sponar, R. P. Harti, J. S. White, J. Kohlbrecher, W. Treimer, and C. Regg for the fruitful discussions. We thank M. Morgano, J. Hovind, M. Schild, U. Filges, and P. Hautle for technical support. The work is funded by the Swiss National Science Foundation, Project No. 162582, and the Korean-Swiss Science and Technology Program, Project No. EG-KR-1192017.

*Corresponding author markus.strobl@psi.ch

[1] V. W. Cohen, N. R. Corngold, and N. F. Ramsey, Magnetic moment of the neutron, Phys. Rev. 104, 283 (1956).

[2] H. Rauch, A. Zeilinger, G. Badurek, A. Wilfing, W. Bauspiess, and U. Bonse, Verification of coherent spinor rotation of fermions, Phys. Lett. 54A, 425 (1975).

[3] G. Badurek, H. Rauch, A. Zeilinger, W. Bauspiess, and U. Bonse, Phase-shift and spin-rotation phenomena in neutron interferometry, Phys. Rev. D 14, 1177 (1976).

[4] G. L. Greene, N. F. Ramsey, W. Mampe, J. M. Pendlebury, K. Smith, W. B. Dress, P. D. Miller, and P. Perrin, Measurement of the neutron magnetic moment, Phys. Rev. D 20, 2139 (1979).

[5] W. G. Williams, Polarized Neutrons (Clarendon Press, Oxford, 1988).

[6] M. Steiner and K. Siemensmeyer, Nuclear magnetism and neutrons, in Neutron Scattering - Magnetic and Quantum Phenomena, edited by F. Fernandez-Alonso and D. L. Price
(Elsevier, Academic Press, Cambridge, 2015), Vol. 48, pp. 435-488, https://doi.org/10.1016/B978-0-12-802049-4 .00007-5.

[7] C. Rüegg, N. Cavadini, A. Furrer, H. U. Güdel, K. Krämer, H. Mutka, A. Wildes, K. Habicht, and P. Vorderwisch, BoseEinstein condensation of the triplet states in the magnetic insulator $\mathrm{TlCuCl} 3$, Nature (London) 423, 62 (2003).

[8] B. Lake, Soliton chirality: Quantum magnets show their hand, Nat. Phys. 1, 143 (2005).

[9] N. Kardjilov, I. Manke, M. Strobl, A. A. Hilger, W. Treimer, M. Meissner, T. Krist, and J. Banhart, Three-dimensional imaging of magnetic fields with polarized neutrons, Nat. Phys. 4, 399 (2008).

[10] M. Kenzelmann, T. Strässle, C. Niedermayer, M. Sigrist, B. Padmanabhan, M. Zolliker, A. D. Bianchi, R. Movshovich, E. D. Bauer, J. L. Sarrao, and J. D. Thompson, Coupled superconducting and magnetic order in CeCoIn5, Science 321, 1652 (2008).

[11] D. J. Morris, D. A. Tennant, S. A. Grigera, B. Klemke, C. Castelnovo, R. Moessner, C. Czternasty, M. Meissner, K. C. Rule, J. U. Hoffmann, K. Kiefer, S. Gerischer, D. Slobinsky, and R.S. Perry, Dirac strings and magnetic monopoles in the spin ice Dy2Ti 2O7, Science 326, 411 (2009).

[12] P. Merchant, B. Normand, K. W. Krämer, M. Boehm, D. F. McMorrow, and C. Rüegg, Quantum and classical criticality in a dimerized quantum antiferromagnet, Nat. Phys. 10, 373 (2014).

[13] N. Leo, V. Carolus, J. S. White, M. Kenzelmann, M. Hudl, P. Tolédano, T. Honda, T. Kimura, S. A. Ivanov, M. Weil, T. Lottermoser, D. Meier, and M. Fiebig, Magnetoelectric inversion of domain patterns, Nature (London) 560, 466 (2018).

[14] D. Sarenac, J. Nsofini, I. Hincks, M. Arif, C. W. Clark, D. G. Cory, M. G. Huber, and D. A. Pushin, Methods for preparation and detection of neutron spin-orbit states, New J. Phys. 20, 103012 (2018).

[15] S. Mühlbauer, D. Honecker, É. A. Périgo, F. Bergner, S. Disch, A. Heinemann, S. Erokhin, D. Berkov, C. Leighton, M. R. Eskildsen, and A. Michels, Magnetic smallangle neutron scattering, Rev. Mod. Phys. 91, 015004 (2019).

[16] J. Shen, S. J. Kuhn, R. M. Dalgliesh, V. O. de Haan, N. Geerits, A. A. M. Irfan, F. Li, S. Lu, S. R. Parnell, J. Plomp, A. A. van Well, A. Washington, D. V. Baxter, G. Ortiz, W. M. Snow, and R. Pynn, Unveiling contextual realities by microscopically entangling a neutron, Nat. Commun. 11, 930 (2020).

[17] J. Valsecchi, J. S. White, M. Bartkowiak, W. Treimer, Y. Kim, S. W. Lee, D. M. Gokhfeld, R. P. Harti, M. Morgano, M. Strobl, and C. Grünzweig, Visualization of compensating currents in type-II/1 superconductor via high field cooling, Appl. Phys. Lett. 116, 192602 (2020).

[18] M. Strobl, W. Treimer, P. Walter, S. Keil, and I. Manke, Magnetic field induced differential neutron phase contrast imaging, Appl. Phys. Lett. 91, 254104 (2007).

[19] F. M. Piegsa, B. Van Den Brandt, P. Hautle, J. Kohlbrecher, and J. A. Konter, Quantitative Radiography of Magnetic Fields Using Neutron Spin Phase Imaging, Phys. Rev. Lett. 102, 145501 (2009). 
[20] W. Treimer, O. Ebrahimi, N. Karakas, and R. Prozorov, Polarized neutron imaging and three-dimensional calculation of magnetic flux trapping in bulk of superconductors, Phys. Rev. B 85, 184522 (2012).

[21] W. Treimer, O. Ebrahimi, and N. Karakas, Observation of partial Meissner effect and flux pinning in superconducting lead containing non-superconducting parts, Appl. Phys. Lett. 101, 162603 (2012).

[22] A. S. Tremsin, N. Kardjilov, M. Strobl, I. Manke, M. Dawson, J. B. McPhate, J. V. Vallerga, O. H. W. Siegmund, and W. B. Feller, Imaging of dynamic magnetic fields with spin-polarized neutron beams, New J. Phys. 17, 043047 (2015).

[23] M. Schulz, A. Neubauer, P. Böni, and C. Pfleiderer, Neutron depolarization imaging of the hydrostatic pressure dependence of inhomogeneous ferromagnets, Appl. Phys. Lett. 108, 202402 (2016).

[24] I. Dhiman, R. Ziesche, V. K. Anand, L. Riik, G. Song, A. T. M. N. Islam, I. Tanaka, and W. Treimer, Thermodynamics of Meissner effect and flux pinning behavior in the bulk of single-crystal LaSrCuO, Phys. Rev. B 96, 104517 (2017).

[25] M. Sales, M. Strobl, T. Shinohara, A. Tremsin, L. T. Kuhn, W. R. B. Lionheart, N. M. Desai, A. B. Dahl, and S. Schmidt, Three dimensional polarimetric neutron tomography of magnetic fields, Sci. Rep. 8, 2214 (2018).

[26] A. Hilger, I. Manke, N. Kardjilov, M. Osenberg, H. Markötter, and J. Banhart, Tensorial neutron tomography of three-dimensional magnetic vector fields in bulk materials, Nat. Commun. 9, 4023 (2018).

[27] M. Sales, T. Shinohara, M. K. Sorensen, E. B. Knudsen, A. Tremsin, M. Strobl, and S. Schmidt, Three dimensional polarimetric neutron tomography-Beyond the phasewrapping limit, J. Phys. D 52, 205001 (2019).

[28] P. Jorba, M. Schulz, D. S. Hussey, M. Abir, M. Seifert, V. Tsurkan, A. Loidl, C. Pfleiderer, and B. Khaykovich, High-resolution neutron depolarization microscopy of the ferromagnetic transitions in $\mathrm{Ni} 3 \mathrm{Al}$ and $\mathrm{HgCr} 2 \mathrm{Se} 4$ under pressure, J. Magn. Magn. Mater. 475, 176 (2019).

[29] J. Valsecchi, R. P. Harti, M. Raventós, M. D. Siegwart, M. Morgano, P. Boillat, M. Strobl, P. Hautle, L. Holitzner, U. Filges, W. Treimer, F. M. Piegsa, and C. Grünzweig, Visualization and quantification of inhomogeneous and anisotropic magnetic fields by polarized neutron grating interferometry, Nat. Commun. 10, 3788 (2019).

[30] F. Bloch, Nuclear induction, Phys. Rev. 70, 460 (1946).

[31] M. Strobl, H. Heimonen, S. Schmidt, M. Sales, N. Kardjilov, A. Hilger, I. Manke, T. Shinohara, and J. Valsecchi, Polarization measurements in neutron imaging, J. Phys. D 52, 123001 (2019).

[32] C. Grünzweig, C. David, O. Bunk, M. Dierolf, G. Frei, G. Kühne, J. Kohlbrecher, R. Schäfer, P. Lejcek, H. M. Rønnow, and F. Pfeiffer, Neutron Decoherence Imaging for Visualizing Bulk Magnetic Domain Structures, Phys. Rev. Lett. 101, 025504 (2008).

[33] I. Manke, N. Kardjilov, R. Schäfer, A. Hilger, M. Strobl, M. Dawson, C. Grünzweig, G. Behr, M. Hentschel, C. David, A. Kupsch, A. Lange, and J. Banhart, Three-dimensional imaging of magnetic domains, Nat. Commun. 1, 125 (2010).
[34] S. W. Lee, K. Y. Kim, O. Y. Kwon, N. Kardjilov, M. Dawson, A. Hilger, and I. Manke, Observation of magnetic domains in insulation-coated electrical steels by neutron dark-field imaging, Appl. Phys. Express 3, 106602 (2010).

[35] T. Reimann, S. Mühlbauer, M. Schulz, B. Betz, A. Kaestner, V. Pipich, P. Böni, and C. Grünzweig, Visualizing the morphology of vortex lattice domains in a bulk type-II superconductor, Nat. Commun. 6, 8813 (2015).

[36] B. Betz, P. Rauscher, R. P. Harti, R. Schäfer, A. IrastorzaLanda, H. Van Swygenhoven, A. Kaestner, J. Hovind, E. Pomjakushina, E. Lehmann, and C. Grünzweig, Magnetization response of the bulk and supplementary magnetic domain structure in high-permeability steel laminations visualized in Situ by neutron dark-field imaging, Phys. Rev. Applied 6, 024023 (2016).

[37] T. Reimann, M. Schulz, D. F. Mildner, M. Bleuel, A. Brûlet, R. P. Harti, G. Benka, A. Bauer, P. Böni, and S. Mühlbauer, Domain formation in the type-II/ 1 superconductor niobium: Interplay of pinning, geometry, and attractive vortex-vortex interaction, Phys. Rev. B 96, 144506 (2017).

[38] R. P. Harti, M. Strobl, R. Schäfer, N. Kardjilov, A. S. Tremsin, and C. Grünzweig, Dynamic volume magnetic domain wall imaging in grain oriented electrical steel at power frequencies with accumulative high-frame rate neutron dark-field imaging, Sci. Rep. 8, 15754 (2018).

[39] A. Backs, M. Schulz, V. Pipich, M. Kleinhans, P. Böni, and S. Mühlbauer, Universal behavior of the intermediate mixed state domain formation in superconducting niobium, Phys. Rev. B 100, 064503 (2019).

[40] M. Strobl, C. Grünzweig, A. Hilger, I. Manke, N. Kardjilov, C. David, and F. Pfeiffer, Neutron Dark-Field Tomography, Phys. Rev. Lett. 101, 123902 (2008).

[41] M. Strobl, General solution for quantitative dark-field contrast imaging with grating interferometers, Sci. Rep. 4, 7243 (2015).

[42] A. Momose, H. Takano, Y. Wu, K. Hashimoto, T. S. M. Hoshino, Y. Seki, and T. Shinohara, Recent progress in $\mathrm{X}$-ray and neutron phase imaging with gratings, Quantum Beam Sci. 4, 9 (2020).

[43] F. Pfeiffer, C. Grünzweig, O. Bunk, G. Frei, E. Lehmann, and C. David, Neutron Phase Imaging and Tomography, Phys. Rev. Lett. 96, 215505 (2006).

[44] J. Valsecchi, M. Strobl, R. P. Harti, C. Carminati, P. Trtik, A. Kaestner, C. Grünzweig, Z. Wang, K. Jefimovs, and M. Kagias, Characterization of oriented microstructures through anisotropic small-angle scattering by 2D neutron dark-field imaging, Commun. Phys. 3, 1 (2020).

[45] W. Just, C. S. Schneider, R. Ciszewski, and C. G. Shull, Refraction of thermal neutrons by shaped magnetic fields, Phys. Rev. B 7, 4142 (1973).

[46] G. Badurek, H. Rauch, A. Wilfing, U. Bonse, and W. Graeff, A perfect-crystal neutron polarizer as an application of magnetic prism refraction, J. Appl. Crystallogr. 12, 186 (1979).

[47] M. Morgano, S. Peetermans, E. Lehmann, T. Panzner, and U. Filges, Neutron imaging options at the BOA beamline at Paul Scherrer Institut, Nucl. Instrum. Methods Phys. Res., Sect. A 754, 46 (2014).

[48] W. Treimer, O. Ebrahimi, N. Karakas, and S. O. Seidel, PONTO-An instrument for imaging with polarized 
neutrons, Nucl. Instrum. Methods Phys. Res., Sect. A 651, 53 (2011).

[49] J. Valsecchi and R. P. Harti, Ngimagic/Tapy: Tapy 0.2, Tapy 0.2 , https://doi.org/https://dx.doi.org/10.5281/ZENODO.803047 (2017).

[50] See the Supplemental Material, which includes Refs. [51-60], at http://link.aps.org/supplemental/10.1103/ PhysRevLett.126.070401 for the experimental situation through the formalism of Wigner quasi-distribution functions.

[51] W. Gerlach and O. Stern, Der experimentelle Nachweis der Richtungsquantelung im Magnetfeld, Z. Phys. 9, 349 (1922).

[52] J. E. Sherwood, T. E. Stephenson, and S. Bernstein, Sterngerlach experiment on polarized neutrons, Phys. Rev. 96, 1546 (1954).

[53] H. G. Hasler and G. Weber, Ein Stern-Gerlach-Versuch mit Neutronen, Atomkernenergie 7, 170 (1962).
[54] T. J. Jones and W. G. Williams, A Stern-Gerlach polarimeter for cold neutrons, J. Phys. E. 13, 227 (1980).

[55] B. Alefeld, G. Badurek, and H. Rauch, Longitudinal SternGerlach effect with slow neutrons, Phys. Lett. 83A, 32 (1981).

[56] R. Golub, R. Gähler, and T. Keller, A plane wave approach to particle beam magnetic resonance, Am. J. Phys. 62, 779 (1994).

[57] F. Mezei, Lect. Notes Phys. (Springer, Berlin, Heidelberg, Germany, 1979), Vol. 128, pp. 3-26.

[58] E. Wigner, On the quantum correction for thermodynamic equilibrium, Phys. Rev. 40, 749 (1932).

[59] L. Mandel and E. Wolf, Opt. Coherence Quantum Opt. (Cambridge University Press, Cambridge, England, 1995).

[60] G. Badurek, H. Rauch, M. Suda, and H. Weinfurter, Identification of nonclassical states in neutron spin precession experiments, Opt. Commun. 179, 13 (2000). 